Chubb, S.R. Framework for Understanding LENR Processes, Using Conventional Condensed Matter Physics. in Eleventh International Conference on Condensed Matter Nuclear Science. 2004. Marseille, France.

\title{
Framework for Understanding LENR Processes, Using Conventional Condensed Matter Physics
}

\author{
Scott R. Chubb \\ Research Systems, Inc., 9822 Pebble Weigh Ct., Burke VA 22015-3378, USA, chubbscott@aol.com
}

\begin{abstract}
Conventional Condensed Matter physics provides a unifying framework for understanding Low Energy Nuclear Reactions (LENR's) in solids. In the paper, standard many-body physics techniques are used to illustrate this fact. Specifically, the paper shows that formally the theories by Schwinger, Hagelstein, and Chubb and Chubb (C\&C), all can be related to a common set of equations, associated with reaction rate and energy transfer, through a standard many-body physics procedure (R-Matrix theory). In each case, particular forms of coherence are used that implicitly provide a mechanism for understanding how LENR's can proceed without the emission of high energy particles. In addition, additional ideas, associated with Conventional Condensed Matter physics, are used to extend the earlier Ion Band State (IBS) model by C\&C. The general model clarifies the origin of coherent processes that initiate LENR's, through the onset of ion conduction that can occur through ionic fluctuations in nanoscale crystals. In the case of $\mathrm{PdD}_{\mathrm{x}}$, these fluctuations begin to occur as $\mathrm{x} \rightarrow 1$ in sub-lattice structures with characteristic dimensions of $60 \mathrm{~nm}$. The resulting LENR's are triggered by the polarization between injected d's and electrons (immediately above the Fermi energy) that takes place in finite-size PdD crystals. During the prolonged charging of $\operatorname{PdD}_{\mathrm{x}}$, the applied, external electric field induces these fluctuations through a form of Zener tunneling that mimics the kind of tunneling, predicted by Zener, that is responsible for possible conduction (referred to as Zener-electric breakdown) in insulators. But because the fluctuations are ionic, and they occur in $\mathrm{PdD}$, nano-scale structures , a more appropriate characterization is Zener-ionic breakdown in nano-crystalline PdD. Using the underlying dynamics, it is possible to relate triggering times that are required for the initiation of the effect, to crystal size and externally applied fields.
\end{abstract}

\section{INTRODUCTION}

An important challenge for theory is to explain why high energy particles are almost never emitted in LENR's. In fact, through wave-like effects that can occur in condensed matter systems, it is possible for large amounts of momentum to be transferred instantly to distant locations, without requiring that any particular particle (or particles) acquire high velocity. In [1,2], I provided a context for explaining how this can occur through a combination of structural effects (related to crystalline order) and dynamical changes (resulting from momentum fluxes of charged particles) at the boundaries of ordered regions, and through a phenomenon (Broken Gauge Symmetry), in which many particles coherently move, at once. Specifically, in [1,2], I identified a particular Gauge Symmetry (the invariance of the ground state, in bulk regions, which are electronically neutral, with respect to rigid translations that do not alter the separations between charged particles) that is the basis of Mossbauer-like processes, in which a solid, as a whole, can recoil in response to an outside force. The associated Broken Gauge Symmetry (BGS) refers to the fact that near surfaces and interfaces, where charge can accumulate, a preferential gauge, and zero of momentum, is required for each charged particle. In [1], I explicitly identified features associated with the electronic structure of fully-loaded Palladium $(\mathrm{Pd})$ Deuteride $\left(\mathrm{PdD}_{\mathrm{x}}, \mathrm{x} \rightarrow 1\right)$ that explain how this can occur. In particular, the electronic states in PdD, in the immediate vicinity of the Fermi Level, are delocalized and have small overlap with the octahedral site region locations of the Dnuclei (deuterons d's).

Because of this fact, in finite, PdD crystals, outside forces are always needed in order to sustain high loading, and slight variations in loading are required to induce long-range coupling, through vibrational modes that have conventionally been thought to involve acoustical phonons. In fact, in the limit of small variations in loading, these vibrations, are not conventional phonons because the associated coupling does not conserve the number of d's that are present in the crystal. Instead, in the extreme lowtemperature limit, a more appropriate starting point is the Ion Band State (IBS) picture[4] in which each $\mathrm{d}$ effectively occupies a delocalized state, similar to the delocalized (Energy Band) states that electrons 
occupy in ordered solids. In [2,3], I use a generalization of a well-known procedure (Multiple Scattering Theory) in order to explain how the initial IBS theory[4] is related to a more complete description of the nuclear physics problem and to situations in which disorder is present.

In [1], I generalized these results to finite size crystals, with real boundaries. In fact, the essential physics of these papers[1-3], which involves identifying relative time-scales associated with particular transient, resonant processes, is really quite general: it can be re-expressed using standard many-body physics techniques (for example, conventional R-Matrix Theory[5]). Within this context, it is possible to identify relationships between particular theoretical approaches ( Hagelstein's "Unified Phonon-Coupled SU(N) Model/Resonant Group Method"[6], Schwinger's "Nuclear Energy in an Atomic Lattice," coherent phonon model[7], for example) that involve resonant processes and the IBS[1,4] model.

In the next section, I examine this relationship and the importance of certain features (for example, boundaries) that can be included within the more general approach[1-3] that are not included in [6] and [7]. GMST can be used to identify particular forms of coherence and to assess their impact (based on reaction rate and overlap with externally applied fields) on potential dynamical processes. In particular, during the overcharging of portions of a sub-lattice of PdD, using GMST, it can be shown that the fastest, most coherent, dynamical reactions do not alter the charge in the interior (bulk-like) regions of the associated structure. This can occur when the momentum from a particular LENR is transferred directly to the center-of-mass of the particles in the sub-lattice, in a manner that mimics the recoil of a solid in response to the absorption of a gamma ray, in the Mossbauer effect.

Because the associated process does not alter the relative energies or momenta of any of the charges in the "bulk-like" neutral region, it does not alter the ionic charge appreciably, in these regions. (Here, the bulk region refers to the portion of the sub-lattice, where, on the average, no net charge is allowed to accumulate in each unit cell.) Instead, the process induces variations in the ionic charge at the immediate locations of potential reactions and in surface/interface regions, outside the bulk region. (Technically, since a net accumulation of charge is allowed to take place at the locations of potential reactions, as well as at other locations near surfaces and interfaces, these regions are located outside the "bulk region.")

This can occur in a particular limit in which each d occupies the same (lowest energy) IBS, initially, and, effectively, with respect to the remaining (non-IBS) dynamical changes (associated with charge transport from electrons) that result from the presence of an applied electric field, as a function of wavevector, all of the (lowest energy band) IBS's share a common energy eigenvalue. In this limit, as a function of time, all IBS eigenvalues, effectively, are degenerate and will remain degenerate with respect to order-preserving processes. These processes provide a way for the momentum of any collision or reaction in the entire sub-lattice to be shifted, rigidly, to its center-of-mass in response to externally applied fields. The kinds of order-preserving processes that can allow this to occur are referred to as Umklapp Processes or U-Processes.

Beginning from the ground state (GS) of an ordered sub-lattice, involving a finite number of atoms, processes that preserve order can only be initiated, provided the initial and final states differ by specific amounts, and provided the amount of momentum that is imparted does not exceed a particular, maximum value. Because, in the presence of an externally applied field $\vec{E}$, the momentum of each particle with charge $\mathrm{e}$ is shifted, as a function of time $\mathrm{t}$, by an amount $e \vec{E} \mathrm{t}$, the requirement that specific values of momentum are associated with greatest coherence is equivalent to requiring that, at particular times, resonant (Umklapp) processes take place. The additional constraint, that the change in energy associated with the process be so low that the particles in the bulk region of the solid remain in their ground state (GS), further restricts how energy and momentum can be transferred during the associated processes.

The physics of the associated limit essentially is the same physics that describes the onset of conductivity in insulators, through Zener-electric breakdown[8], in which electrons in a filled electron band are subjected to electric fields for such a long time that they effectively tunnel into the first excited state band, where they then, effectively, move (and conduct electricity) through the normal transport processes associated with conventional band theory. Three important differences are: In the PdD situation, 1 . The conduction involves ions, not electrons, 2. Instead of dealing with a filled band, all d's occupy the same state, but this state, effectively, always has vanishingly small dispersion (so that the ions in the state do not create a current because the energy of the state is not altered by changes in the momentum), and 3. The effect is initiated in nano-crystalline structures of PdD (as opposed to macroscopically large insulators).

In the final section, I explain the physics of the associated limit, and its implications. This includes a plausible explanation for the frequently observed (but poorly understood) requirement that the onset of 
an LENR in the PdD system does not occur at the time that high loading is achieved. In particular, a finite incubation (or triggering) time is required that can vary between minutes, hours, days, and (in some cases) even weeks. The triggering time requirement involves crystal size: Beginning with $60 \mathrm{~nm}$ nanocrystalline structures, LENR's are initiated with triggering times that are on the order of minutes through ionic fluctuations (associated with Zener-ionic breakdown); in micron size crystalline structures, the required triggering times (through Zener-ionic breakdown) are on the order of 10's of hours.

\section{FRAMEWORK FOR UNDERSTANDING LENR BASED ON MANY-BODY PHYSICS}

A standard many-body physics technique, R-matrix theory[5], can be employed to understand the relationship between several theories that have been proposed as explanations of LENR's. In particular, in R-matrix theory, the effects of a particular interaction are introduced by dividing all of the (possible) many-body configurations into two sets, associated, respectively, with: 1 . States that involve physically measurable quantities (involving a restricted set of energies and momenta); and 2. The remaining states. Then, by construction, the states from the first set (for example, the GS and the low-lying excitations of the solid) are assumed to play a direct role in the associated dynamics and are relatively well-known; while states from the second set are assumed to play a secondary role and, as a consequence, are not required to be as well characterized. (In practice, in LENR theories, particular models, based on "plausible" assumptions, have been used to model the states associated with the second set.) The assumption that the states from the first set play a direct role means that a restricted form of interaction can be used to model the relevant dynamics, in which both the initial and final states are restricted to have components associated with this particular set of states. Formally, this constraint can be imposed by projecting both the initial and final states onto a subspace, defined by the first set of states. Thus, if $\mid Q_{i}>$ is one of the states in the first set, the average value of a particular operator $O$ is assumed, by construction, to be the average of $P_{Q} O P_{Q}$, where $P_{Q}$ is the projection operator, defined by by

$$
P_{Q} \equiv \sum_{i}\left|Q_{i}\right\rangle\left\langle Q_{i}\right|
$$

Similarly, if $\left|\mathrm{P}_{\mathrm{i}}\right\rangle$ is one of the states in the second set, the associated projection operator $P_{P}$ is defined by

$$
P_{P} \equiv \sum_{i}\left|P_{i}\right\rangle\left\langle P_{i}\right| \equiv 1-P_{Q}
$$

where, implicitly, in a formal sense, it is assumed that the many-body states are complete. In practice, because only a limited amount of information is known about the second set of states associated with Eq. 2 , by construction, normalization and energy conservation are not imposed from the outset in particular regions of space, or as a function of time, but are determined dynamically (as in the renormalization procedure, used in quantum field theory).

As a consequence, an effective, non-Hermitean, energy-dependent many-body Hamiltonian $\mathrm{H}_{\mathrm{eff}}$ can be constructed that describes the relevant dynamics associated with the propagation of states within the subspace defined by $P_{Q}$. In fact, because the momentum/energy cut-off for states that belong in the first or second sets is not unique, the resulting decomposition is not unique. However, neither the loss of uniqueness, or the fact that the resulting Hamiltonian is not Hermitean or energy-independent detracts from its usefulness in assessing the potential importance of coherent effects( in particular) in solids, or, more generally, in the problem of estimating relevant physical quantities. In particular, R-matrix theory can be used to identify key effects associated with the relevant dynamics in many problems in which a dominant form of interaction can be identified.

Consistent with the associated picture, the formal generalization that is used in R-matrix theory involves allowing the energy, associated with solutions of the many-body Schroedinger equation, to be complex (by allowing the wave function to grow or be dampened through dissipative processes that break time-reversal invariance). In R-matrix theory, a particularly useful representation is constructed by requiring that the deviations from perfect time-reversal invariance be infinitesimally small.

The construction is also useful because when causal relationships are imposed, it provides a way to derive an approximate Hamiltonian by "removing" the degrees of freedom of the true Schroedinger equation that are not involved in the associated process through an "inversion procedure." This is accomplished by constructing an effective inverse of the Schroedinger equation, which is used to eliminate the dependence of the equation on the variables (and the associated range of energies) that are not directly involved in the problem. This becomes possible when the energy is allowed to take on complex values. 
In particular, in the "inverted" form of the Schroedinger equation, the effects of the implicit perturbations from states that are not physically important (designated by $\mid \mathrm{P}_{\mathrm{i}}>$ in Eq. 2) on the remaining (physically important) states are inferred through a projection procedure. Formally, this is accomplished through a two step process, in which: 1 . An effective inverse, $G(Z) \equiv \frac{1}{Z-H}$, of the many-body

Schroedinger equation (defined by $\mathrm{H}$ ), associated with a complex value of the energy, $\mathrm{Z}$, is constructed (or assumed), based on a knowledge of the relevant dynamics, initially, and 2. The fact that only a restricted set of states (the states associated with Eq. 1) are important is used to justify eliminating the states that are not included in this set from the relevant dynamics. In particular, if $G(Z)$ is an inverse of the Schroedinger equation, formally, it follows that

$$
G(Z)(Z-H)=1=\left(P_{Q}+P_{P}\right) G(Z)\left(P_{Q}+P_{P}\right)(Z-H)\left(P_{Q}+P_{P}\right)
$$

But then because $P_{Q} P_{P} \equiv 0$, by construction, from Eq.3, it also follows that

$$
\left[P_{Q}(Z-H) P_{Q}-P_{Q} V_{F} P_{P}\left(\frac{1}{Z-H}\right) P_{P} V_{F} P_{Q}\right] P_{Q} G(z) P_{Q}=1
$$

where $\mathrm{V}_{\mathrm{F}}=\mathrm{V}-\mathrm{P}_{\mathrm{Q}} \mathrm{VP}_{\mathrm{Q}}$ is the difference between the full, many-body potential $(\mathrm{V})$ and the projection of $\mathrm{V}$ onto the subspace, defined by $\mid \mathrm{Q}>$, and $P_{Q}(Z-H) P_{P}=-P_{Q} V_{F} P_{P}$ is the negative of the projection of all of the matrix elements that couple the physically realizable states $(\mid \mathrm{Q}>)$ to the remaining $(\mid \mathrm{P}>)$ states.

Eqs. 2 and 3 define an effective Hamiltonian $\mathrm{H}_{\text {eff }}$ (through the inverse, $\mathrm{Z}-\mathrm{H}_{\text {eff }}$, of the projection of $\mathrm{G}(\mathrm{z})$ onto the initial and final states associated with the physically measurable states, $\mid \mathrm{Q}>$ ):

$$
Z-H_{e f f}=P_{Q}(Z-H) P_{Q}-P_{Q} V_{F} P_{P}\left(\frac{1}{Z-H}\right) P_{P} V_{F} P_{Q}
$$

In particular, the effects of the outside perturbations associated with the excited states enter through the additional term, $-P_{Q} V_{F} P_{P}\left(\frac{1}{Z-H}\right) P_{P} V_{F} P_{Q}$.

Eqs.1-5 apply to arbitrary many-body configurations. In problems related to reaction, the relationship between $V$ and the initial state potential $V_{o}$ has to be specified. Provided the final state that results from some outside interaction and the initial state have the same total energy E, all deviations from the initial state involve states that are orthogonal to it. Then, if the initial state can be modeled, using the set of states $\mid \mathrm{Q}>$, the full potential projected onto this set of states must equal $\mathrm{V}_{\mathrm{o}}=\mathrm{P}_{\mathrm{Q}} \mathrm{VP}_{\mathrm{Q}}$, and $\mathrm{V}_{\mathrm{F}}=\mathrm{V}-\mathrm{V}_{\mathrm{o}}=\Delta \mathrm{V}$ is the change in potential that is responsible for the transition to the final state. Because $P_{Q} P_{P}=P_{P} P_{Q}=0$, $\mathrm{P}_{\mathrm{Q}} \mathrm{V}_{\mathrm{F}} \mathrm{P}_{\mathrm{P}}=\mathrm{P}_{\mathrm{Q}} \Delta \mathrm{V} \mathrm{P}_{\mathrm{P}}=\mathrm{P}_{\mathrm{Q}} \Delta \mathrm{V}\left(\mathrm{P}_{\mathrm{P}}+\mathrm{P}_{\mathrm{Q}}\right)=\mathrm{P}_{\mathrm{Q}} \Delta \mathrm{V}$; and similarly, $\mathrm{V}_{\mathrm{F}} \mathrm{P}_{\mathrm{Q}}=\Delta \mathrm{V} \mathrm{P}_{\mathrm{Q}}$. As a consequence, it follows from Eq. 4 that

$$
Z-H_{e f f}=P_{Q}(Z-H) P_{Q}-P_{Q} \Delta V\left(\frac{1}{Z-H}\right) \Delta V P_{Q}=P_{Q}\left(Z-H_{o}-\Delta V\left(\frac{1}{Z-H}\right) \Delta V\right) P_{Q},
$$

where $\mathrm{H}_{\mathrm{o}}=\mathrm{T}+\mathrm{V}_{\mathrm{o}}$ is the initial state, unperturbed Hamiltonian, defined by the kinetic energy $\mathrm{T}$ and initial state potential $\mathrm{V}_{\mathrm{o}}$.

To be consistent with time dependent perturbation theory, since $\mathrm{E}$ is real, $\mathrm{Z}$ can differ from $\mathrm{E}$ only through the addition of a small positive or negative imaginary component (i.e., $Z=E \pm i \delta$ ). As a consequence, it is possible to write two effective Hamiltonians, $H_{\text {eff }}^{+}$and $H_{e f f}^{-}$, which, respectively, describe situations in which coupling through the perturbation $\Delta \mathrm{V}$ (to the states associated with $\mathrm{P}_{\mathrm{P}}$ ) vanishes in the distant past, or future:

$$
H_{e f f}^{ \pm}=P_{Q}\left(H_{o}+\Delta V\left(\frac{1}{E-(H \pm i \delta)}\right) \Delta V\right) P_{Q}
$$

Since it is assumed that the initial state is spanned by the set of states $\mid \mathrm{Q}>, H_{e f f}^{ \pm}$can be understood to be the projection of the full Hamiltonian $\mathrm{H}$ onto a particular set of states, possessing specific boundary conditions and/or that is defined in a particular (restricted) region of space. Because $\mathrm{Z}$ is a complex number, particle flux, state normalization and energy need not be conserved. Implicitly, through the second term (associated with $\mathrm{G}(\mathrm{Z})$ ) in Eq. 7, $H_{\text {eff }}^{ \pm}$includes coupling with "forbidden" states (or "regions"), associated with overlap of the physical system with the sub-space defined by $\mathrm{P}_{\mathrm{P}}$, that is not included in the initial ("allowed") sub-space, defined by $\mathrm{P}_{\mathrm{Q}}$. Consistent with conventions used in formal scattering theory and the Fermi Golden Rule, the imaginary part of the expectation value of the 
difference between the Hamiltonians, ( $\left.\operatorname{Im} a g\left\langle\Psi\left|\left(H_{e f f}^{+}-H_{e f f}^{-}\right)\right| \Psi\right\rangle\right)$, defined by Eq. 7, is interpreted as defining an effective lifetime $\tau$ and rate of interaction $R \equiv \frac{1}{\tau}=\frac{1}{\hbar} \operatorname{Im} a g\left(H_{\text {eff }}^{+}-H_{\text {eff }}^{-}\right)$associated with overlap between the initial states (defined by $\mathrm{P}_{\mathrm{Q}}$ ) with the "forbidden" states (defined by $\mathrm{P}_{\mathrm{P}}$ ) which, by construction, have vanishing overlap with the initial states, in the absence of any perturbation.

In particular, from Eq. 7, it follows that if $\Psi_{\mathrm{o}}$ is any many-body state that is initialized, in the absence of outside perturbations (so that $\mathrm{P}_{\mathrm{Q}} \Psi_{\mathrm{o}}=\Psi_{\mathrm{o}}$ ), the associated values of $\mathrm{R}$ and $\tau$ are given by

$$
\begin{aligned}
R \equiv \frac{1}{\tau} & =\frac{2 \pi}{\hbar}<\Psi_{o}|\Delta V \delta(E-H) \Delta V| \Psi_{o}> \\
& \equiv \frac{2 \pi}{\hbar} \sum_{F} \delta\left(E-E_{\text {exact }}\left(C_{F}\right)\right)\left|<\Psi_{o}\right| \Delta V\left|\Psi_{\text {exact }}\left(C_{F}\right)>\right|^{2},(8)
\end{aligned}
$$

where $\Psi_{\text {exact }}\left(C_{F}\right)$ is any many-body state, possessing a particular configuration (denoted by $\mathrm{C}_{\mathrm{F}}$ ) of particles that solves the Schroedinger equation of the exact Hamiltonian, $\mathrm{H}$, and $\mathrm{E}_{\text {exact }}\left(\mathrm{C}_{\mathrm{F}}\right)$ is its energy.

Three important points are: 1 . Eq. 8 , in principle, is exact; 2. As a consequence, it holds for arbitrary perturbations $\Delta \mathrm{V}$ (and not merely when the $\Delta \mathrm{V}$ is sufficiently small, which is the case, when the Fermi Golden Rule applies); and 3. It is virtually impossible to evaluate the R.S. of Eq. 8 without detailed knowledge either of $\Psi_{\text {exact }}\left(C_{F}\right)$ and/or both $\Psi_{\text {exact }}\left(C_{F}\right)$ and $\Delta \mathrm{V}$. Nonetheless, it is possible to make considerable progress, using Eq.8- 37, by making reasonable assumptions about the relevant physics, associated with the more refined LENR theories. In particular, it is possible to show directly from Eq. 8 (as discussed below) how forms of coherence associated with both BGS or phonons can lead to LENR's.

In applying Eq. 8 to LENR's, two obvious effects have to be explained: 1. The lack of high energy particles that are released in any of the final states; and 2. How to "overcome" the Coulomb barrier. But as opposed to situations in conventional nuclear physics where the initial state involves two particles that collide at a particular location, in a solid, the initial many-body state consists of many particles that potentially can interact with each other at many different locations. Similarly, as opposed to the conventional nuclear physics situation in which a small number of particles appear in the final state, in solids, many particles can interact with each other, instantly. For this reason, the idea of overcoming a Coulomb barrier at a particular location, in which a particle tunnels across a region where its kinetic energy becomes forbidden classically, can be replaced by a more general concept, in which many particles interact through one, two, or even many "forbidden" transitions (associated, potentially, with many different possible configurations $C_{F}$ ) in which many particles can interact with each other at many locations.

In the idealized limit in which many particles are allowed to respond coherently, for example, through a particular transition, involving a particular, common characteristic frequency, $v$, the total energy associated with the response, in principle, can be as large as the product of $\hbar v$ with the total number $\left(\mathrm{N}_{\mathrm{T}}\right)$ of particles. As a consequence, in the most efficient transitions, intuitively, one expects that characteristic rates $\mathrm{R}$ associated with coherent many-body processes, in principle, can become proportional to $\mathrm{N}_{\mathrm{T}}$, which means that it can become possible to achieve the kinds of reaction rates (and lifetimes) associated with conventional nuclear reactions, even when the energies associated with the particular transition of an individual particle are quite small.

For this reason, Schwinger[7], Hagelstein[6], and Chubb[1] invoked arguments associated either with Eq. 8, or (in Hagelstein's case) to an equivalent formulation, based on an alternative formalism (the Resonant Group Method), involving coupling to a coherent many-body state. Unfortunately, before his death, Schwinger did not complete his model. However, he made approximate calculations of R, by evaluating Eq. 8, directly, based on the assumption that all of the available energy from the nuclear reaction was released coherently by producing a large number of phonons, associated with a particular frequency. Also, as opposed to using wave functions associated with conventional tunneling at a particular location, he dealt with the overlap problem by assuming that the deuterons could be constrained by the lattice to occupy different locations within a common unit cell, where conceivably, they could have appreciable overlap. However, although he based his overlap argument on assumptions that appeared to involve a realistic geometry[9] (in which d's simultaneously occupy the tetrahedral and octahedral sites in a particular unit cell), subsequently, this geometry was shown to be in-appropriate. Also, in Schwinger's treatment, the associated coherence involved coupling through phonons, derived within the harmonic approximation, which, if applicable, only applies at low loading. 
Subsequently, Hagelstein developed a considerably more sophisticated treatment that also is based on the idea of coherent phonons. As opposed to assuming that in the complete the many-body configuration (associated with $\Psi_{\text {exact }}\left(C_{F}\right)$ ) the overlap occur between deuterons exclusively in different lattice site locations (which was assumed by Schwinger), or at the same site, Hagelstein considered both situations. He found by including coupling to a phonon spectrum that is dominated by a single, coherent phonon, through the associated "forbidden states," which result when the displacements associated with the phonons are included in the behavior of the deuteron-deuteron separation variable, that appreciable overlap between deuterons only takes place when the two deuterons occupy the same site. (Schwinger undoubtedly would have found a similar result had he not been misled by the incorrect geometry suggested by Sun and Tomanek[9].) In his treatment, Hagelstein[6] explicitly includes the coordinate dependencies of all of the nucleons in the problem, as well as an effective (Gamow-like) tunneling factor that impedes deuteron-deuteron overlap when two deuterons occupy the same site.

He also finds that by applying second (and/or higher) order perturbation theory (which effectively can be inferred to all orders, using Eq. 8), it is possible (through phonon exchange) to derive appreciable reaction rates, associated with "fast alpha" particle creation (i.e., where the alpha energies exceed 10 $\mathrm{MeV}$ ), by allowing for a pair of deuterons on one site to interact with a second pair, located on a different site, through phonon exchange. In order to obtain appreciable reaction rates for "slow alpha" production, he concludes it is necessary to include additional, non-linear phonon coupling in the associated, siteother-site, deuteron pair interactions. He also points out a potentially important, implicit form of additional interaction could enhance the non-linear coupling: the fact that because phonons are bosons, effectively, a collection of coherent phonons could stimulate additional, coherent phonons (that possess the same frequency), through stimulated emission processes (analogous to the kinds of processes that are used in Lasers to produce coherent beams of light). Hagelstein speculates that amplification of phonon exchange from such processes could make it possible for many units of angular momentum to be transferred between the coherent phonons and pairs of deuterons through the associated coupling.

In fact, as pointed out above and elsewhere[1], in the particular limit, near full-loading, in finite size crystals of PdD, the extreme long wavelength, acoustical phonons that result from small variations in deuterium loading, actually are deuteron ion band states (IBS's). In a real solid, these variations in loading couple directly to the electric (and ionic) charge in the surface region. Thus, implicitly they provide long-range coupling that is required to be coherent (since deuterons are bosons, on length scales associated with electromagnetic interaction).

Because in the limit of small additional loading, the associated "vibrations" lead to small fluctuations in deuteron charge in the immediate vicinity of deuterons (located in the octahedral sites) that have been loaded into the lattice, the associated IBS's , effectively, appear to be the phonons that couple most directly to deuterons in the lattice. But the associated IBS's involve coupling that leads to an effective, external electric field (resulting from build-up and depletion of charge in non-bulk regions). In the context of an idealized form of Eq. 8, involving an initial state consisting of the many-body state associated with the GS of an infinitely-periodic, neutral, Harmonic crystal, the effects of coupling to a many-body state that includes IBS's occur through changes in the electrostatic zero of energy of each charged particle (which effectively alters the chemical potentials of the "conventional" phonons and electrons, associated with the periodic solid) and through non-linear (second and higher order) processes.

As a consequence, although the coupling to IBS's occurs through variations in charge that "appear" to be acoustical phonons in finite PdD crystals, relative to the initial state associated with the infinite crystal, case, these variations "appear" to be optical phonons. Thus, in the context of Hagelstein's model, a case can be made that the "coherent" phonons that he uses could be IBS's, in certain circumstances. On the other hand, at elevated temperatures, as a result of electron-IBS coupling, and electron-phonon coupling, again through Eq. 8, occupied and/or unoccupied IBS's will induce additional coherent and incoherent phonons, that also could play a role in his model.

Regardless of whether or not Hagelstein's model applies, there are more direct forms of coherence, associated with BGS[1,10], and IBS's, that can lead to nuclear interactions, especially, either at lower temperatures, or in smaller (nano-scale) crystals (or both). In particular, (as noted above) GMST, in its most general form, is equivalent to R-matrix theory. To establish this, it is sufficient to: 1. Identify $\mid \mathrm{Q}>$ with the ground state, $V_{G S} \equiv P_{Q} V P_{Q}$, and, 2. For computing the time evolution of the overlap of $\Psi_{G S}$ with an arbitrary (excited state) $\Psi^{\prime}$, in the bulk region, formally, to require that $\mathrm{V}^{\prime}=\mathrm{P}_{\mathrm{P}} \mathrm{VP}_{\mathrm{P}}$. Thus, it follows, that the difference in potential $\Delta \mathrm{V}=-\left(\mathrm{V}-\mathrm{V}^{\prime}\right)$. Then, each matrix element in Eq. 8 can be expressed in terms of the overlap of $\Psi_{\mathrm{GS}}$ and $\Psi$ ' with the divergence of the velocity flux $\mathrm{v}(\mathrm{r})$, used in $[1,2]$ : 


$$
\frac{\partial<\Psi^{\prime}\left|\Psi_{G s}\right\rangle}{\partial t}=\iiint d^{3} r_{1} \ldots d^{3} r_{n} \frac{\partial\left(\Psi^{* *} \Psi_{G S}\right)}{\partial t}=-\int d^{3} r \nabla \cdot\left\langle\Psi^{\prime}|v(r)| \Psi_{G S}\right\rangle+\left\langle\Psi^{\prime}\left|\frac{V_{G S}-V^{\prime}}{i \hbar}\right| \Psi_{G S}\right\rangle=0,
$$

where $\left\langle\Psi^{\prime}|v(r)| \Psi_{G S}\right\rangle=\sum_{j} \iiint d^{3} r_{1} . d^{3} r_{n} \delta^{3}\left(r-r_{j}\right) \frac{1}{m_{j}}\left(\frac{\hbar}{2 i}\left[\Psi^{\prime} * \nabla_{r_{j}} \Psi_{G S}-\nabla_{r_{j}} \Psi^{\prime} * \Psi_{G S}\right]-\frac{e_{j}}{c} \Psi^{\prime} * A_{e f f}\left(r_{j}\right) \psi_{G S}\right)$, and

$\mathrm{A}_{\mathrm{ef}(\mathrm{r}}(\mathrm{r})=\left(\mathrm{A}(\mathrm{r})+\mathrm{A}^{\prime}(\mathrm{r})\right) / 2$ is the arithmetic mean between the vector potential $\mathrm{A}^{\prime}(\mathrm{r})$ associated with the state $\Psi$ ' and the comparable vector potential A(r), associated with the state $\Psi_{\mathrm{GS}}$. GMST follows from the observation that Eq. 9 applies, in general terms, for any two states that have the same total energy, globally, but different potential energies.

The problem of applying GMST, in its complete form (as in Eq. 9), is formidable because, in principle, it provides a solution to the general many-body problem. In the limit in which particular symmetries are present, however, GMST can be extremely useful. In particular, as I have noted previously [8,11], in order to have minimal overlap with outside processes, in particular regions of space, the flux contributions from $\int d^{3} r \nabla^{\bullet}\left\langle\Psi^{\prime}|v(r)| \Psi_{G S}>\right.$ from these regions are required to vanish.

Because of this fact, implicit forms of symmetry can evolve that preserve the overlap (or lack of overlap) between many-body configurations.

In situations involving finite solids, with real boundaries, an important source of symmetry results from a form of Galilean relativity that exists because of approximate invariance (of the bulk region) with respect to rigid, Galilean transformations. In the absence of interaction with surface regions and lattice imperfections, the associated symmetry makes it impossible to distinguish the GS from other states that can be derived from the GS by simply shifting the zero of momentum of the solid because such a shift can be accounted for through a trivial gauge transformation (in which a constant is added to $A_{\mathrm{ef}(}(\mathrm{r})$ ).

Broken Gauge Symmetry (BGS) in finite crystals occurs because charge accumulates in surface/interface regions. In the case of LENR's, BGS can also occur in regions where overlap between charged particles can take place. Appreciable overlap can also take place through BGS, between charged particles in solids, because $\frac{\hbar}{2 i}\left[\Psi^{\prime} * \nabla_{r_{j}} \Psi_{G S}-\nabla_{r_{j}} \Psi^{\prime *} \Psi_{G S}\right]$ and $\left.\frac{e_{j}}{c} \Psi^{\prime *} A_{e f f}\left(r_{j}\right) \psi_{G S}\right)$ locally can change suddenly, without the velocity v(r), ever becoming large. Potentially, the most relevant nuclear-scale processes (that occur in situations involving BGS) are initiated when the bulk region, effectively remains in its GS, and momentum is transferred coherently, through rigid translations (associated with UmKlapp processes) that preserve periodic order from locations where overlap between nuclei can take place (and the associated ionic charge from IBS's can accumulate) to regions outside the bulk region. Within this context, initially the "forbidden" regions are the non-bulk regions. Through subsequent transfer of momentum to these regions, heating is triggered. As a consequence, nuclear products (heat and new elements) are expected to appear outside the bulk.

In order to describe how these processes couple to IBS's, in situations involving BGS, it is necessary to relate the individual coordinates $\left(r_{1}, \ldots ., r_{N_{T}}\right)$ of the various particles in the many-body wave function to the coordinate $R_{c m}$ associated with the center-of-mass, the separation variables $r_{i j}$ that describe the difference in the position of each particle from the remaining particles, and the total mass $\mathrm{M}$ of the collection of particles. In particular, by definition,

$$
r_{i j}=r_{i}-r_{j}=-r_{j i} ; \quad R_{c m}=\frac{\sum_{i=1, N_{T}} m_{i} r_{i}}{M}
$$

and, from Eq. 10, it follows that

$$
r_{1}=\sum_{j=2, N_{T}} \frac{m_{j} r_{1 j}}{M}+R_{c m} ; \quad r_{j}=r_{1}-r_{1 j} \quad(j \neq 1)
$$

The summation in Eq.11, in principle, should be carried out over all particles in the many-body system. In fact, the only contributions that are relevant in any reaction occur from particles that have non-vanishing flux, at the boundaries of the bulk region[1]. Also, because at the immediate location of a potential nuclear reaction, a change in charge can take place that can alter the charge and raise the energy of the system, technically, the associated location is not part of the GS, bulk system (since a net flux of charge can occur, as a result of the reaction), even when the associated redistribution occurs in a nuclearscale size volume, surrounding the reaction, where the potential is altered. In any case, potential nuclear reactions can occur whenever the coordinate $\mathrm{r}_{\mathrm{i}}$ associated with the center of mass of a single deuteron (d) 
in the many-body wave function, that possesses a wave-like, IBS form (in the bulk), has overlap either with a second $d$ or with some other nucleus, that possesses a coordinate $r_{j}$. This overlap can only occur at locations where $r_{i j}$ can become sufficiently small that the strong force becomes appreciable (which occurs in the idealized limit in which, on the scale of electromagnetic interaction, $\mathrm{r}_{\mathrm{ij}} \rightarrow 0$ ).

The lowest energy forms of LENR, within the context of BGS, involve situations in which the reaction is triggered by potential overlap between an initial state involving p's, t's, or d's in IBS form. In the vicinity of a location where this can occur, as a result of Coulomb repulsion, the potential energy is required to change rapidly. As a consequence, a surface integral contribution occurs in Eq. 9, at each location where $r_{i j} \rightarrow 0$, as a result of the flux of particles (and charge) that is introduced as a result of the change in potential the location of a nuclear reaction. In particular, as in [1] it follows that we may write,

$$
\begin{aligned}
\left\langle\Psi^{\prime}\left|\frac{V-V^{\prime}}{i \hbar}\right| \Psi\right\rangle & =\int d^{3} r \nabla \bullet\left\langle\Psi^{\prime}|v(r)| \Psi\right\rangle=\int_{\text {bulk boundary }} d^{2} S \quad \hat{n} \bullet\left\langle\Psi^{\prime}|v(r)| \Psi\right\rangle \\
& =\sum_{j,\left\{d_{j}\right\}} \iiint d^{3\left(N_{T}-1\right)} r \int_{S_{d_{j}}} d^{2} S_{d_{j}} n_{d_{j}} \cdot \frac{1}{m_{j}}\left(\frac{\hbar}{2 i}\left[\Psi^{\prime *} \nabla_{r_{j}} \Psi-\nabla_{r_{j}} \Psi^{*} \Psi\right]-\frac{e_{j}}{c} \Psi^{\prime *} A_{e f f}\left(r_{j}\right) \Psi\right) .
\end{aligned}
$$

Here (in the first line), we have assumed that V-V' vanishes in the bulk region, and we have used Greens theorem to convert the divergence of the velocity (which, in principle, includes all particles that leave or enter the bulk) into a surface integral that extends over the boundary of the bulk (which we have represented through the label beneath the final integral, in the first line, and through the two dimensional integration symbol that appears in the same integral ). In the second line of this integral, we have divided this final integral into domains, which we have denoted, using the symbol $d_{j}$, and through the label $S_{d_{j}}$, which refers to the area of the associated domain. In particular, each of these domains is associated with an effective boundary of a location (defined by $\mathrm{r}_{\mathrm{ij}} \rightarrow 0$ ) where a nuclear reaction can occur, or to the external boundary of the bulk region, which occurs at the boundary of the region where, on the average, a net accumulation of charge begins to take place in each unit cell. Also, in this integral, the multi-dimensional integral ( $\iiint d^{3\left(N_{T}-1\right)} r$ ) extends over the remaining coordinates. It follows from Eqs. 10 and 12 that energy and momentum can be transferred directly from nuclear reactions, in the interior (non-bulk) regions to locations outside the solid. Provided the final and initial state (nuclear) portions of the wave function involve (different) stable, ground state configurations (for example, when the initial and final states are ground states of different nuclei), when the number $(N)$ of locations where potential reactions take place is sufficiently large, it is possible to derive an effective velocity flux (through an expansion of the bulk wave function, in terms of the shift in the zero of energy), across the (nuclear region) boundaries in Eq.12, based on the semi-classical equations of motion[1].

In the most coherent situation, no excitation of any state, whatsoever, in the bulk region can take place; while outside the bulk region, momentum can be transferred from the solid as a whole into surface/interface regions (through center-of-mass motion and excitation and the resulting ion-electron interaction), or into the bulk region, from nuclear reactions, in the interior. But since

$$
\nabla_{r_{1}}=\left(\sum_{j=2, N_{T}} \nabla_{r_{1 j}}+\frac{m_{1}}{M} \nabla_{R_{c m}}\right) ; \quad \nabla_{r_{j}}=-\nabla_{r_{1 j}}+\frac{m_{j}}{M} \nabla_{R_{c m}},
$$

at locations where $r_{i, j} \rightarrow 0$, in the surface/interface region effective discontinuities in $\nabla_{r_{j}} \Psi$ and $\nabla_{r_{j}} \Psi^{\prime *}$ (wave function cusps) can occur (associated with sudden changes in potential), either through ion-ion or electron-ion overlap. In Eq. 12, these effects can be treated by enclosing each location associated with a cusp with a volume of size $V_{M T}$. In principle, size $V_{M T}$ can be a small, enclosing sphere, surrounding the singularity, which (in analogy with the situation in multiple-scattering theory) we will refer to as a muffin tin (or MT). Globally, we could pick each MT to be a sphere of nuclear dimension. On the other hand, in the surface and interface regions, where significant electron-ion interaction takes place, d's and/or ${ }^{4} \mathrm{He}$ nuclei become partially neutralized. In this limit, the lifetime of each d-reactant and/or ${ }^{4} \mathrm{He}$-product nucleus in a particular IBS can be reduced dramatically. As a consequence, a more useful representation of the associated perturbation, in analogy with situations in which electrons become important, involves allowing for the possibility that the (residual) singularity (if it exists at all) be allowed to extend over a larger domain (which asymptotically can approach an atomic scale characteristic size). In the associated interaction, coupling to final state many-body states can occur in which ${ }^{4} \mathrm{He}$ either appears in a localized 
(ionic or partially ionic) form at MT locations, or delocalized, in a surface IBS form. The most coherent effects occur when a large number of states, in the bulk, that are related to each other, through rigid, Galilean transformations, become approximately degenerate.

Previously[1], by requiring that the overlap between states near the GS, with each other, and with the GS, be stable with respect to variations in the zeroes of energy and momentum, I generalized a number of results associated with conventional energy band theory, as it relates to charged particles in infinitelyrepeating ordered solids to finite size solids, with real boundaries. By requiring that the net force on the bulk region vanish and that the potential and density decay (in surface regions) sufficiently slowly, I was able to generalize the semi-classical equations of energy band theory (for infinite lattices) to situations involving finite-size lattices. Essentially, the associated construction (which is a form of BGS) is equivalent to identifying the preferential gauge that minimizes coupling between low-lying states (associated with different wave-vectors) in the bulk with each other and with the GS. In general, because the basis of the BGS is the idea that all of the particles in the bulk can "move" rigidly, in response to some symmetry-breaking perturbation, the associated effect involves a form of coherence in momentum that can be large at particular locations but be distributed, instantly, to many locations. For this reason, through BGS, for example, the velocity operator (in Eq. 9), which can become discontinuous, can change suddenly, but without high energy particles ever being emitted. This kind of effect is guaranteed to be possible in LENR's provided the target nucleus either occupies or could occupy an IBS in bulk regions (which requires that it be a proton [p], a triton [t], or a d). Alternatively, potential LENR's could be initiated outside the bulk, with an alternative target nucleus, through some alternative effect involving accumulation of charge (for example, in surface regions), triggered by the bulk, through an Umklapp process. (This kind of reaction could also trigger "fast alpha" production, as well as some of the other phenomena, suggested by Hagelstein.) For illustrative purposes, however, in describing the most coherent form of LENR, we will focus exclusively on the $\mathrm{d}+\mathrm{d} \rightarrow{ }^{4} \mathrm{He}$ reaction.

\section{TRIGGERING TIME AND COHERENCE THROUGH ZENER-IONIC BREAKDOWN}

BGS occurs when, as a result of a continuous symmetry, many energy states, resulting from many energy configurations, become approximately degenerate. In the case of d's in PdD, the greatest degeneracy occurs in an extreme limit in which, effectively, initially, all of the d's have the same energy, for all values of the momentum. In $\operatorname{PdD}_{\mathrm{x}}$, in finite regions, where $\mathrm{x} \rightarrow 1$, the presence of an electric field $\vec{E}$ can induce long-range fluctuations (in x) that induce variations $\vec{E}_{\text {ind }}$ in $\vec{E}$ that cause the degeneracy to be lifted. The resulting form of coupling is strongest when the contribution to the (ionic) current from two d's that occupy IBS's becomes vanishingly small. The associated interaction (which, in its strongest form, can mimic the phenomena of Bloch Oscillations in insulators) can trigger $\mathrm{d}+\mathrm{d} \rightarrow{ }^{4} \mathrm{He}$ reactions, with minimal coupling to the bulk (and with no high energy particles), provided the crystal size is sufficiently large, and the initial state d's interact with each other and with the applied $\vec{E}$-field for a sufficiently long period of time. The initiation of $d+d \rightarrow{ }^{4} H e$ LENR's, through the most coherent processes, occurs in a limit that involves the transfer of small amounts of charge from (cusp) regions throughout the interior (where it is highly polarized), to surface/interface regions through a process that initially, effectively, excites d's in the lowest energy band (which is effectively dispersionless ) to a higher energy band. The underlying physical effect is analogous to a phenomenon (Zener-electric breakdown[8] in insulators) that was first postulated by Zener (based on an idea by Bloch) that could lead to conduction in insulators. In particular, Bloch suggested that the semi-classical equations describe how the energy and momentum of "quasi-particles" (electrons) change, as a function of time. He observed that in the limit of a one-dimensional periodic lattice, in the absence of collisions, a particular phenomenon (referred to as a Bloch oscillation) might take place. In particular, when the lattice is onedimensional, it can be represented in terms of integral multiples of a single primitive vector (the lattice spacing a). Then, if the precise wave function associated with the conduction electrons could be written in terms of a superposition of band state energy wave functions, in the long wave-length limit (where the semi-classical equations apply), in the absence of magnetic fields, the associated charge would appear to oscillate (as a function of wave-vector and time) between the boundaries of the First Brillouin zone, with a period $\tau$ of oscillation defined by the applied electric field $\vec{E}$, through the relationship,

$$
e \vec{E} \tau=\frac{2 \pi \hbar}{a}
$$


Zener pointed out [8] that because $\vec{E}$ can become quite large in insulators and/or semi-conductors, the idealized (semi-classical) limit, associated with a single band, can breakdown, implicitly, through excitations that lead to inter-band transitions. Instead, he pointed out that implicitly these forms of excitations could be treated by allowing for the possibility that the associated charge could tunnel into different (higher energy) bands, where alternative forms of transport (including those associated with the semi-classical theory) could apply.

In general, an externally applied $\vec{E}$-field alters the zero of momentum of the bulk region, as a result of polarizing the charged particles in non-bulk regions. The associated polarization is strongest in the surface/interface regions and occurs as a result of accumulation of charge. Typically, in transition metals, screening occurs very rapidly (within 5-10 layers from a surface or interface). Thus, for example, in most situations, an applied voltage of 1 volt across the entire solid induces an $\vec{E}$-field that varies between $\sim 0.05$ and $\sim 0.025$ volts per Angstrom, in the immediate vicinity of surfaces/interfaces. On the other hand, because, in order to sustain high-loading, it is always necessary to apply an external field, small fluctuations of charge, associated with deviations from perfect stoichiometry, involve non-local coupling that extends over many unit cells. Relative to the perfectly stoichiometric PdD compound, these fluctuations can be represented (for example, using R-matrix theory) through an external perturbation $\Delta \mathrm{V}$ that alters the vector potential (and zero of momentum), in the regions (associated with cusps) where d-d overlap can occur.

The most coherent forms of reaction occur in the limit in which the current becomes vanishingly small. In particular, in order for the bulk region to remain in its GS, the possible energy (or momentum) release, throughout the bulk region must be less than the characteristic energy or momentum release that is associated with processes that would excite conventional (optical) phonons (associated with the bulk, in the idealized limit of an infinite PdD lattice). In particular, because the smallest (zero-point) energies associated with optical phonons in PdD are typically $\leq \sim 0.01 \mathrm{eV}$, this requirement can be used to identify a useful bound for the (dominant) contributions to potential reactions associated with the interior (cusp regions) from the time-dependent terms that result from the change in electric field $\Delta \vec{E}=\vec{E}-\vec{E}_{\text {ind }}$, through the associated induced perturbation to the Hamiltonian (through the vector potential $\Delta \vec{A}=-\Delta \vec{E} c t)$. In particular, when the associated change in energy is $\int_{n o n-b u l k} d^{3} x \Delta \vec{E} t\left\langle\Psi|J(x)| \Psi_{G S}\right\rangle \leq$ $\sim 0.01 \mathrm{eV}$, an appropriate bound for $\mathrm{J}$ is

$$
\int_{n o n-b u l k} d^{3} x\left\langle\Psi|J(x)| \Psi_{G S}\right\rangle \leq \sim \frac{0.01 e V}{|\Delta \vec{E}| t} \text {. }
$$

On the other hand, because the bulk region is required to remain in its ground state, the shift in the zero of momentum is equivalent to a rigid, Galilean transformation, in which beginning from a state, associated with vanishing IBS current (which occurs when all d's occupy the lowest energy k=0 IBS), the wave-vectors of all d's that have overlap in the interior cusp regions are shifted coherently by an amount $e \Delta \vec{E} t$. But this is only allowed provided the resulting wave-vector $\vec{k}(t)=e \Delta \vec{E} t / \hbar$ of the single IBS (associated with all d's in the cusp region) remains in the First Brillouin Zone, defined either by the entire bulk region, or by a (Bravais) sub-lattice of the bulk. With increasing time, for a fixed value of $\Delta \vec{E}$, in principle, the bulk region can still remain approximately in its GS (in the absence of nuclear reactions), even when $\vec{k}(t)$ does not remain in the first Brillouin Zone. Specifically, a particular discrete set of values of the time $t=t(\vec{i})$ exists that define situations where the shift in the wave-vector zero, $t(\vec{i}) \frac{e \Delta \vec{E}}{\hbar}$, identically equals a reciprocal lattice vector. (The vector symbol $\vec{i}$ is used as a short-hand for the three numbers, $i_{1}, i_{2}$, and $i_{3}\left\{\vec{i} \equiv\left(i_{1}, i_{2}, i_{3}\right)\right\}$ that are used to construct the reciprocal lattice.) The possible values of $t(\vec{i})$ can be constructed from discrete units of time, $\Delta t_{\alpha}(\alpha=1,3)$, using the primitive vectors $\vec{b}_{j}(\mathrm{j}=1,3)$ that define the Bravais Lattice[12]:

$$
t=t(\vec{i}) \equiv t\left(i_{\alpha}\right) \equiv i_{\alpha} \Delta t_{\alpha} \quad \alpha=1,2,3, \Delta t_{\alpha}=\frac{2 \pi \hbar}{\left(e \vec{b}_{\alpha} \cdot \Delta \vec{E}\right)} .
$$

At each of the values of $\mathrm{t}$, in Eq. 15, a resonant, Umklapp process can occur in which, the bulk, or a sub-lattice of the bulk can effectively undergo a rigid, Galilean translation. It also follows that in the most coherent situation, where the shift in zero is coherently added to each d, since the total shift is

$$
N \vec{k}(t(\vec{i}))=N e \Delta \vec{E} t(\vec{i}) / \hbar \quad(N=\text { Number of Unit Cells })
$$


and an Umklapp process also occurs at additional times, defined by

$$
t=t\left(\frac{i_{\alpha}}{N}\right)=\frac{i_{\alpha} \Delta t_{\alpha}}{N}
$$

At alternative times, (where $t$ can never be represented, using Eqs. 15-17), especially in smaller lattices, the associated shifts in momentum zero alter the distributions of charge in the non-bulk regions, which, in turn, alter the value of $\Delta \vec{E}$. Provided $N$ is sufficiently small, the associated phenomena can be viewed, effectively, as elastic d-d scattering processes, in which momentum, associated with d-d collisions (in cusp regions) in the interior, is transferred to the surface/interface regions, without altering the bulk. As $N$ becomes sufficiently large that the total shift in zero of momentum becomes comparable to the amount that would normally be associated with the gamma ray that is released in the conventional $\mathrm{d}+\mathrm{d} \rightarrow{ }^{4} \mathrm{He}+\gamma$ reaction, new forms of collisions can take place (in cusp regions), involving a final state in which a net flux of ${ }^{4} \mathrm{He}$ is transferred through an IBS current in the interior cusp region, and either through contributions from the terms that involve currents (and vector potentials) or through the dipoles (and $\vec{E}$ fields), to MT volume cusp regions in the vicinity of the surface and/or interface. Key effects that play a role in triggering are associated with the relevant timescales, and the flux of (positive and negative) charges into and away from the surface/interface region.

The initiation of $d+d \rightarrow{ }^{4} \mathrm{He}$ LENR, through the most coherent processes, which occurs in the limit of small current and long time, involves small amounts of charge that are initially highly polarized (in cusp regions) throughout the interior, followed by a small transfer of charge through a process that initially, effectively, excites d's in a particular band to a higher band. Thus, the triggering process, in the most coherent regime, is analogous to the transition between an insulating and conducting state that is driven by increasing periodic order, as a function of time. An important distinction between this transition and Zener-elcctronic breakdown, however, is that in the situation in fully-loaded PdD, the transition involves ions (as opposed to electrons). For this reason, as opposed to Zener-electronic breakdown, a more appropriate name for the phenomenon might be Zener-ionic breakdown.

In [1], I estimated a minimal crystal size (of $\sim 3000$ unit cells), associated with the onset of the most coherent process. The characteristic dimension of the associated crystal is $\sim 6 \mathrm{~nm}$. I based this estimate on the fact that in the absence of any accumulation of charge in the cusp regions, asymptotically, it is possible to equate the change in momentum (defined implicitly by $N e \Delta \vec{E} t(\vec{i}) / \hbar$ in Eq.16) with the total momentum that the bulk would have in a reference frame that moves with the common velocity that cusp and bulk regions would have in the limit in which all regions move rigidly. Because this estimate fails to include the asymmetry associated with the applied fields, it has no time-scale associated with it, or preferential orientation. Crystal size, orientation (with respect to the fields) and lattice imperfections all are important in triggering the process.

In the idealization of a perfect bulk region, surrounded by a uniform surface region, the lowest energy configurations minimize stress and strain along the surface/bulk boundary. This suggests that in such an environment, the induced electric field (from Coulombic repulsion/nuclear reaction in interior, cusp regions) will be oriented in opposition to the applied field. But detailed calculations, based on a microscopic model of the nuclear physics and atomic/ionic/electronic structure, would be required to understand the magnitude and orientation of the induced response. Historically, it has been observed that the time required to trigger $d+d \rightarrow{ }^{4}$ He varies between several weeks, to several hours, or (for example, in laser-triggering experiments[13]) to several minutes, or less. Zener-ionic breakdown provides a potential mechanism for understanding how such a wide variability in triggering time $t$ can occur. In particular, using Eqs. 15-17, it is possible to relate crystal size to information about the values of $t$, and the magnitude of $\Delta \vec{E}$. Eq. 14 can be used to relate $\Delta \vec{E}$ to the magnitude of the IBS current. Consistent with the associated picture is the idea that although the nuclear reaction would occur over some nanoscale size region of the lattice, in order to preserve periodic order, charge neutralization in the MT volumes, associated with cusp regions outside the bulk, would extend considerably further beyond the boundary of the nano-scale region, through an effective damping of the associated induced dipoles. Within this context, it is reasonable to assume that the induced charge polarization would involve effective values of $\Delta \vec{E}$ that are also consistent with values of $\mathrm{t}_{\text {trig }} \sim 10-10^{4}$.

For illustrative purposes, consider a situation in which the induced potential difference (during the triggering process) is $\sim 1$ Volt and that it extends over a cubic structure, possessing a characteristic dimension of $1 \mathrm{~cm}$. In a situation in which the polarization in charge is approximately the same everywhere in the non-bulk, surface and interface region (which includes all of the cubic structure, 
except for the nano-scale bulk region where the transfer of momentum occurs from the nuclear processs), nuclear reaction effectively induces a value of $\Delta \vec{E} \approx 10^{-8} \mathrm{~V} / \stackrel{o}{A}=1 \mathrm{~V} / \mathrm{cm}$. When $N_{\text {vert }}=1.5 \times 10$ (as in the bound, involving $6 \mathrm{~nm}$ characteristic crystal dimension, identified in [1]), using Eq. 15 (as in the derivation of Eq. 63) it follows that $\Delta t_{1} i_{1}=\Delta t_{2} i_{2}=\frac{4 \pi N_{\text {vert }}}{a|e \Delta \vec{E}|}=\mathrm{t}_{\text {trig }}=3.03 \mu \mathrm{s}$; while if $N_{\text {vert }}=1.5 \times 100000$ (which applies to a sub-lattice having characteristic dimension of $\sim 60 \mu \mathrm{m}$ ), $\mathrm{t}_{\text {trig }} \sim 840$ Hours. In the first example $\left(N_{v e r t}=1.5 \times 10, \mathrm{t}_{\text {trig }} \sim 3 \mu s\right), \frac{1}{\hbar}\left|\frac{\partial \varepsilon}{\partial \vec{k}}\right| \leq \sim \frac{0.01 \mathrm{eV}}{|e \Delta \vec{E}| t_{\text {trig }}}=3.30 \times 10^{12} \frac{\stackrel{o}{\mathrm{~A}}}{\mathrm{~s}}$, which is roughly an order of magnitude larger than the characteristic group speed associated with IBS's that have appreciable dispersion (and charge transport). In the second example, $\left(N_{\text {vert }}=1.5 \times 100000, \mathrm{t}_{\text {trig }} \sim 840\right.$ Hours $)$, the associated value of $\frac{1}{\hbar}\left|\frac{\partial \varepsilon}{\partial \vec{k}}\right| \leq \sim 0.330 \frac{\stackrel{A}{A}}{S}$ is more than 12 orders of magnitude smaller than the characteristic value, associated with a lattice that has appreciable IBS current. The transition between IBS currentconducting to IBS-insulating crystalline structures occurs when $\frac{1}{\hbar}\left|\frac{\partial \varepsilon}{\partial \vec{k}}\right| \leq \sim 3.30 \times 10^{9} \frac{\stackrel{o}{A}}{\mathrm{~s}}$. This corresponds to $N_{\text {vert }}=150$, which applies when the crystal has a characteristic dimension of $\sim 60 \mathrm{~nm}$.

\section{ACKNOWLEDGEMENT}

I would like to thank Talbot Chubb, David Nagel, and Mitchell Swartz for providing valuable suggestions about the material in this paper.

\section{REFERENCES}

[1]S.R. Chubb, Proc. ICCF10 (in press) . http://www.lenr-canr.org/acrobat/ChubbSRnutsandbol.pdf

[2] S.R. Chubb, Trans. Amer. Nuc. Soc. 88,618 (2003).

[3]S.R. Chubb and T.A. Chubb, Proc. ICCF8, 385 (2000). http://www.lenrcanr.org/acrobat/ChubbSRtheoretica.pdf

[4] T.A. Chubb and S.R. Chubb, Fus. Tech. 17, 710 (1990). S.R. Chubb and T.A. Chubb, AIP Conf Proc. 228, Provo, UT, 691 (1991).

[5]Burke P G and Berrington K A 1993 Atomic and Molecular Processes: an R-matrix Approach (Bristol: IOP Publishing)

[6] P. Hagelstein, "Unified Phonon-Coupled SU(N) Models for Anomalies in Metal Deuterides," Proc. ICCF10 (in press). http://www.lenr-canr.org/acrobat/Hagelsteinunifiedpho.pdf

[7] Schwinger, J., Proc. The First Annual Conference on Cold Fusion. 1990, p. 130-136.

[8] C. Zener Proc. R. Soc. A 145523 (1934).

[9] Z. Sum and D. Tomanek, Phys. Rev. Lett. 63, 59 (1989).

[10] Chubb, S.R. and T.A. Chubb. Proc ICCF9, 57-62 (2002); http://www.lenrcanr.org/acrobat/ChubbSRrelationsh.pdf. P.W. Anderson, Basic Notions of Condensed Matter Physics. ( Benjamin-Cummings Pub. Co., Menlo Park, CA, 1984), Chap 1, pp 8-69.

[11]S.R. Chubb, "Impact of Boundary Effects Involving Broken Gauge Symmetry on LENR's," Proc . ICCF10 (in press). Also, http://www.lenr-canr.org/acrobat/ChubbSRimpactofbo [12]N.W.Ashcroft and N.D. Mermin, Solid State Physics. (Saunders College Publishing, Orlando, FL, 1976),pp 64-82.

[13] Cravens, D.G. and D.J. Letts, Proc. ICCF10 (in press); also, http://www.lenrcanr.org/acrobat/CravensDpracticalt.pdf. Letts, D.J. and D.G. Cravens, Proc. ICCF10 (in press); also, http://www.lenr-canr.org/acrobat/LettsDlaserstimu.pdf 